\title{
Function of JARID2 in bovines during early embryonic development
}

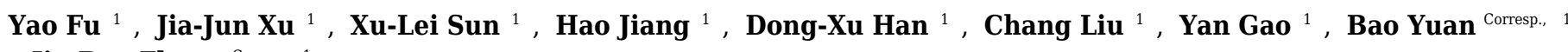
, Jia-Bao Zhang ${ }^{\text {Corresp. } 1}$

1 Department of Laboratory Animals, College of Animal Sciences, Jilin University, Changchun, Jilin, China

Corresponding Authors: Bao Yuan, Jia-Bao Zhang

Email address: yuan_bao@jlu.edu.cn, zjb515@126.com

Histone lysine modifications are important epigenetic modifications in early embryonic development. JARID2, which is a member of the jumonji demethylase protein family, is a regulator of early embryonic development and can regulate mouse development and embryonic stem cell (ESC) differentiation by modifying histone lysines. JARID2 can affect early embryonic development by regulating the methylation level of H3K27me3, which is closely related to normal early embryonic development. To investigate the expression pattern of JARID2 and the effect of JARID2-induced H3K27 methylation in bovine oocytes and early embryonic stages, JARID2 mRNA expression and localization were detected in bovine oocytes and early embryos via qRT-PCR and immunofluorescence in the present study. The results showed that JARID2 is highly expressed in the germinal vesicle (GV), MII, 2-cell, 4-cell, 8-cell, 16-cell and blastocyst stages, but the relative expression level of JARID2 in bovine GV oocytes is significantly lower than that at other oocyte/embryonic stages $(p<0.05)$, and JARID2 is expressed primarily in the nucleus. We next detected the mRNA expression levels of embryonic development-related genes (OCT4,SOX2andc-myc) after JARID2 knockdown through JARID2-2830-siRNA microinjection to investigate the molecularpathwayunderlying the regulation of H3K27me3 byJARID2during earlyembryonic development.Theresultsshowedthattherelativeexpression levels of these genes in 2-cell embryos weresignificantlyhigherthan those in the blastocyststage,and expression levels were significantly increased after JARID2 knockdown. Insummary, the present study identified the expression pattern of JARID2 in bovine oocytes and at each earlyembryonic stage, and the results suggest that JARID2 plays a key role in early embryonic development by regulating theexpression of OCT4, SOX2 and c-myc via modification of H3K27me3expression. This work provides new data for improvements in the efficiency of in vitro embryo culture as well as a theoretical basis for further studying the regulatory mechanisms involved in early embryonic development. 
2 Function of JARID2 in bovines during early embryonic 3 development

5 Yao Fu' ${ }^{1 \dagger}$, Jia-Jun Xu' ${ }^{1 \dagger}$, Xu-Lei Sun${ }^{1}$, Hao Jiang1, Dong-Xu Han', Chang Liu', Yan Gao', 6 Bao Yuan ${ }^{1 *}$ and Jia-Bao Zhang ${ }^{1 *}$

81 Department of Laboratory Animals, College of Animal Sciences, Jilin University, Changchun

9 130062, Jilin, P.R. China

$10 \uparrow$ Yao Fu and Jia-Jun Xu contributed equally to this article.

* Corresponding author:

13 Jia-Bao Zhang

14 Laboratory Animal Center, College of Animal Sciences, Jilin University, 5333 Xi'an Road, 15 Changchun 130062 (P.R. China)

16 Tel./Fax +8643187836551

17 Bao Yuan

18 Laboratory Animal Center, College of Animal Sciences, Jilin University, 5333 Xi'an Road,

19 Changchun 130062, (P.R. China)

Tel./Fax +8643187836536

21 E-mail: zjb515@126.com (J.Zhang), yuan_bao@jlu.edu.cn (B. Yuan) 


\section{ABSTRACT}

Histone lysine modifications are important epigenetic modifications in early embryonic development. JARID2, which is a member of the jumonji demethylase protein family, is a regulator of early embryonic development and can regulate mouse development and embryonic stem cell (ESC) differentiation by modifying histone lysines. JARID2 can affect early embryonic development by regulating the methylation level of $\mathrm{H} 3 \mathrm{~K} 27 \mathrm{me} 3$, which is closely related to normal early embryonic development. To investigate the expression pattern of JARID2 and the effect of JARID2-induced H3K27 methylation in bovine oocytes and early embryonic stages, JARID2 mRNA expression and localization were detected in bovine oocytes and early embryos via qRT-PCR and immunofluorescence in the present study. The results showed that JARID2 is highly expressed in the germinal vesicle (GV), MII, 2-cell, 4-cell, 8-cell, 16-cell and blastocyst stages, but the relative expression level of JARID2 in bovine GV oocytes is significantly lower than that at other oocyte/embryonic stages $(\mathrm{p}<0.05)$, and JARID2 is expressed primarily in the nucleus. We next detected the mRNA expression levels of embryonic development-related genes (OCT4, SOX2 and c-myc) after JARID2 knockdown through JARID2-2830-siRNA microinjection to investigate the molecular pathway underlying the regulation of $\mathrm{H} 3 \mathrm{~K} 27 \mathrm{me} 3$ by JARID2 during early embryonic development. The results showed that the relative expression levels of these genes in 2-cell embryos were significantly higher than those in the blastocyst stage, and expression levels were significantly increased after JARID2 knockdown. In summary, the present study identified the expression pattern of JARID2 in bovine oocytes and at each early embryonic stage, and the results suggest that JARID2 plays a key role in early embryonic development by regulating the expression of OCT4, SOX2 and c-myc via modification of $\mathrm{H} 3 \mathrm{~K} 27 \mathrm{me} 3$ expression. This work provides new data for improvements in the efficiency of in vitro embryo culture as well as a theoretical basis for further studying the regulatory mechanisms involved in early embryonic development. 


\section{INTRODUCTION}

Epigenetic modifications are common biological phenomena involved in gene regulation and are essential for maintaining the normal physiological activities of mammals. Epigenetic modification refers to the heritable modification of gene expression, without any changes in the DNA sequence. Many studies have shown that changes in a variety of epigenetic modifications occur during mammalian embryonic development. Early embryonic development is the period in which cell division and differentiation are most active, and there is large-scale transcription of zygotic genes. At this stage, genes are subject to activation and inhibition under the control of signals from both inside and outside of cells, and many regulatory mechanisms participate in their regulation. Studies conducted in recent years have increasingly shown that epigenetic regulation plays an important role during mammalian embryonic development.

Epigenetic modifications mainly include DNA methylation and histone modification, which generally have a synergistic effect on mammalian gene expression regulation (Delcuve et al. 2009). Acetylation and methylation are the most important factors in histone modification. DNA is methylated via the transfer of a methyl group (CH3) from S-adenosylmethionine (SAM) to cytosine by DNA methyltransferase (Jurkowska et al. 2011). This covalent modification may change gene expression by altering chromatin condensation and heterochromatin formation and generally inhibits gene expression (Brenet et al. 2011; Choy et al. 2010). DNA methylation is critical for the development and reprogramming of mammalian embryos and mainly plays roles in regulating gene expression, genomic imprinting, transposon inactivation, $\mathrm{X}$ chromosome inactivation and alternative splicing of mRNA (Cotton et al. 2011; Dean et al. 2005; Isagawa et al. 2011; Malousi et al. 2008; Verona et al. 2003; Walsh et al. 1998). In general, DNA methylation is negatively correlated with gene expression, and there is a negative correlation between the methylation of a gene and its expression. Histones are an important component of eukaryotic nuclear chromosomes. There are many amino acids in histones that can be covalently modified by reactions including methylation, acetylation, and ubiquitination. These amino acids may cause some local chromatin structures to "open" or close"; they can also determine the 
specific area of DNA to which special proteins can bind. Such modifications can promote normal transcription, reproduction and repair. Acetylation and methylation of histones are the most common and important epigenetic modifications. Histone methylation modifications promote or inhibit gene expression, depending on the site as well as the degree of methylation. Different methylation sites and different degrees of methylation present different functions. In summary, histone methylation-modifying enzymes play an important role in early embryonic development. Histone methylation and demethylation exist in a state of dynamic equilibrium. These processes are catalyzed by histone methyltransferase (HMT) and histone demethylase (HDMT), respectively. Modifications performed by histone methyltransferase can cause the nucleosome to directly adjust the structure of chromatin, thus regulating the expression of related genes.

Histone modification plays an important role in early embryonic development. For example, in mouse oocytes, histones may maintain acetylation until oocyte follicular lysis at the germinal vesicle (GV) stage (Kim et al. 2003a). In sheep oocytes, although no acetylation of H3K9 is detected from the GV stage to the MII stage, high levels of acetylation are detected in male and female pronuclei at the zygote stage, and the degree of acetylation is similar to that in mouse zygotes (Hou et al. 2008). During the maturation of mouse oocytes, H3K9 maintains a constant degree of methylation, while methylation does not appear in the male chromosome group until the first mitosis after fertilization (Yeo et al. 2005). It was found that the degree of H3K27 methylation affects early embryonic development, and the degree of H3K27me3 methylation is closely related to normal early embryonic development (Marinho et al. 2017; Ross et al. 2008; Zhang et al. 2009).

Lysine demethylases, which include the LSD family and the (jumonji C) JmjC domain family, play a role in early embryonic development. The JmjC family includes the most types of methyltransferases, each of which has a JmjC domain; most of these methyltransferases enable histone lysine demethylation using $\mathrm{Fe}(\mathrm{II})$ and $\alpha$-ketoglutarate $(\alpha \mathrm{KG})$ as co-enzyme factors (Jackson et al. 2003). Unlike other families of demethylases, JHDMs can remove all histone lysine methylation moieties. JARID proteins are members of the JmjC methyltransferase family 
105

106

107

108

109

110

111

112

113

114

115

116

117

118

119

120

121

122

123

124

125

126

127

128

129

130

131

and have been widely studied. The JARID2 (jumonji, AT-rich interactive domain 2) protein is one member of the jumonji demethylase protein family; unlike other members in this family, JARID2 may not exhibit demethylase activity (Kooistra \& Helin 2012). JARID2 was first identified in mice as a regulator of neural development (Jung et al. 2005b; Takeuchi et al. 1995); it was shown to interact with most inhibitory complex 2 to regulate the self-renewal ability of embryonic stem cells (Herz \& Shilatifard 2010; Pasini et al. 2010). The absence of JARID2 reduces the transferability of mouse hematopoietic stem cells and embryonic stem cells (ESCs)line 17 (Kinkel et al. 2015). At the molecular level, JARID2 interacts with histone methyltransferases to regulate the expression of target genes (Cai et al. 2013; Kim et al. 2003b; Margueron et al. 2009). JARID2 also plays an important role in the normal development of mouse embryos and the differentiation of ESCs.

However, the epigenetic modifications that occur during early bovine embryonic development are still unclear. Thus, it is important to determine whether JARID2 exerts an effect on bovine embryonic development and determine the mechanism of regulation. This study explores the expression pattern of JARID2 at all bovine oocyte and early embryonic stages and its mechanism of action, including its influence on H3K27 methylation.

\section{MATERIALS AND METHODS}

\section{Ethics statement}

The experiments were performed strictly according to the guidelines of the Guide for the Care and Use of Laboratory Animals of Jilin University. In addition, all experimental pro tocols were approved by the Institutional Animal Care and Use Committee of Jilin Unive rsity (Permit Number: 20160522).

\section{Collection of bovine oocytes and in vitro maturation}

Bovine ovaries were collected from the No. 3 Slaughter House of the Haoyue Group in Changchun City. After collection, they were placed in vacuum bottles containing normal saline at $37^{\circ} \mathrm{C}$ and then sent to the laboratory within $3-5 \mathrm{~h}$. After cleaning the ovaries with normal 
132 saline containing penicillin and streptomycin (Sigma, USA) at $37^{\circ} \mathrm{C}$, follicular fluid was 133 extracted from the 2- to 8-mm normal follicles on the surface of the ovaries and then slowly 134 injected into a 50-ml centrifuge tube. The collected follicular fluid was left undisturbed for 15 135 min to allow the cells to settle at the bottom of the centrifuge tube. The precipitated cells were 136 subsequently extracted and placed in a plate containing washing solution (10\% PVA) (Sigma, 137 USA). The cumulus oocyte complexes (COCs) with more than 3 layers of cumulus cell coats were selected under a microscope, quantified and collected in drops of culture medium for in vitro oocyte maturation; $18-20 \mathrm{COCs}$ were placed in each drop and transferred to a $38.5^{\circ} \mathrm{C}$ incubator with $5 \% \mathrm{CO}_{2}$ for $24 \mathrm{~h}$.

\section{In vitro fertilization}

Frozen semen (Simmental semen from the Yanbian Animal Husbandry Development Group Co., LTD) straws were quick-thawed in a $37^{\circ} \mathrm{C}$ water bath, and the thawed semen was then transferred to equilibrated Dulbecco's phosphate-buffered saline (DPBS). After the gently shaking the semen, it was centrifuged for $3 \mathrm{~min}$ at $1,300 \mathrm{rpm}$; this process was repeated twice. Then, the semen was resuspended and centrifuged for $3 \mathrm{~min}$ at 1,300 $\mathrm{rpm}$. The precipitated semen was transferred to a straw containing equilibrated in vitro fertilization solution, which was subsequently placed in an incubator at $38.5^{\circ} \mathrm{C}$ with $5 \% \mathrm{CO}_{2}$ for $30 \mathrm{~min}$, allowing the sperm to complete the swimming up process. Then, liquid was collected from the upper layers and inspected with a microscope to calculate sperm viability and density. After washing with in vitro fertilization solution, the mature COCs and the sperm acquired after swimming up were transferred to equilibrated fertilization drops; each of these droplets contained 15 COCs, and they were placed in an incubator at $38.5^{\circ} \mathrm{C}$ with $5 \% \mathrm{CO}_{2}$ for fertilization. After $24 \mathrm{~h}$, the cumulus cells were removed using $0.1 \%$ hyaluronidase (Sigma, USA) enzyme solution.

\section{In vitro cultivation}

Fertilized embryos were selected and washed three times with culture medium and then transferred to in vitro cultivation (IVC) drops that were equilibrated in advance for further 
159

160

161

162

163

164

165

166

167

168

169

170

171

172

173

174

175

176

177

178

179

180

181

182

183

184

cultivation. Each drop contained 18-20 fertilized embryos. The embryos were collected at different developmental stages according to the experimental design.

\section{Cell transfection}

Bovine cumulus cells were cultivated in 24-well plates and transfected as follows when cell fusion reached 75\%: based on ratio of $2 \mu \mathrm{l}$ of Lipofectamine ${ }^{\mathrm{TM}} 2000$ (Invitrogen, USA) to $1 \mu 1$ of siRNA (Gene Pharma, China) per well of a plate, $100 \mu \mathrm{l}$ of serum-free DMEM/F12 medium (Gibco, USA) was added to each well. The plate was then incubated at room temperature for 5 min, after which Lipofectamine ${ }^{\mathrm{TM}} 2000$ and the siRNA mixture were added, followed by incubation at room temperature for $20 \mathrm{~min}$. Finally, $200 \mu \mathrm{l}$ of the siRNA-Lipofectamine ${ }^{\mathrm{TM}} 2000$ mixture was added to each well, and the cells were incubated at $37^{\circ} \mathrm{C}$. Eight hours later, the cells were transferred to complete medium containing $10 \%$ fetal bovine serum (BI, Israel) and then collected after $36 \mathrm{~h}$.

\section{Microinjection of siRNA for JARID2 knockdown}

Twenty-four hours after in vitro fertilization, the COCs were treated with $0.1 \%$ hyaluronidase to remove the surrounding cumulus cells. Then, the COCs were washed three times with fresh solution to ensure that all cumulus cells were removed. The zygotes were next randomly divided into two groups and transferred to IVC drops containing CB buffer; the concentration of siRNA was maintained at $10 \mu \mathrm{M}$. After installing the syringe needle and holding pipette, the operating wall was adjusted to start the injection. After microinjection, the zygotes were transferred to IVC drops that were equilibrated in advance; each drop contained 15 zygotes, which were collected at different stages.

\section{Immunofluorescence detection}

Immunofluorescence staining of embryos was performed as follows. Early embryos at different stages were placed in 24-well Petri dishes containing 4\% paraformaldehyde and fixed at room temperature with ventilation for $30 \mathrm{~min}$. After fixing, the embryos were permeabilized with $0.5 \%$ Triton $\mathrm{X}-100$ at $4^{\circ} \mathrm{C}$ for $20 \mathrm{~min}$. Then, the early embryos were transferred to a $1 \%$ BSA 
solution and incubated at $37^{\circ} \mathrm{C}$ for $1 \mathrm{~h}$. The primary antibody (Abcam, UK) was diluted 200 times with a $1 \%$ BSA solution, followed by incubation with the samples overnight at $4{ }^{\circ} \mathrm{C}$. Secondary antibodies (Boster, USA) were also diluted with 1\% BSA solution, followed by incubation in the dark with the samples for $1 \mathrm{~h}$ in the incubator at $37^{\circ} \mathrm{C}$. Next, the samples were incubated with the DAPI (Sigma, USA) fluorescence staining reagent, diluted 1,000 times, 2 min at room temperature. Then, the slides were sealed, and the expression patterns of the target proteins were observed. The fluorescence staining intensity of early embryos at different developmental stages was detected with fluorescence intensity analysis software.

In the abovementioned immunofluorescence staining process, the samples were repeatedly washed at each step.

\section{Immunofluorescence intensity analysis}

Photographs of each experimental group were acquired using the same parameters. The mean fluorescence intensity in the nuclear region was used as the measurement index for changes in H3K27me3. We performed statistical analyses of the mean fluorescence intensity in the early embryonic nuclei at the 2-cell stage, 4-cell stage, 8-cell stage and blastocyst stage in the JARID2 siRNA silencing group and the negative control group, employing Image-Pro Plus 6.0 software.

\section{Statistical analysis}

We analyzed the experimental data from more than 3 independent experiments per group with the analysis of variance (ANOVA) module of SPSS16.0 statistical software. The data are expressed as the mean \pm standard deviation (SD).

\section{RESULTS}

\section{Relative expression and localization of JARID2 in bovine oocytes and early embryos}

To compare the differences in expression between JARID2 bovine oocytes at the GV and MII stages and early embryos at the 2-cell, 4-cell, 8-cell, 16-cell and blastocyst stages, we designed primers for JARID2 and measured the JARID2 expression level with qRT-PCR. The 
212

213

214

215

216

217

218

219

220

221

222

223

224

225

226

227

228

229

230

231

232

233

234

235

236

237

238

experimental results showed that JARID2 was expressed in bovine oocytes at the GV and MII stages and in early embryos at the 2-cell, 4-cell, 8-cell, 16-cell and blastocyst stages. JARID2 expression was lowest in oocytes at the GV stage, where it was significantly lower than that in oocytes/embryos at other stages $(p<0.05)$. There was no significant difference in JARID2 expression among oocytes/embryos at other stages ( $\mathrm{p}>0.05)$ (Fig. 1A).

Immunofluorescent staining was performed on early bovine embryos at the 2-cell, 4-cell, 8cell, 16-cell and blastocyst stages. We found that JARID2 was expressed at all stages of embryonic development and was mainly expressed in the cell nucleus (Fig. 1B).

We measured the fluorescence signals with a fluorescence microscope and collected images using the same parameters. In addition, we determined the mean fluorescence intensity at the embryonic nuclei, to represent the amount of JARID2 expression at different stages of early embryonic development. The mean fluorescence intensity of the embryonic nuclei was measured at the 2-cell, 4-cell, 8-cell, 16-cell and blastula stages with Image-Pro Plus 6.0. The results are shown in Fig. 1C. There was no significant difference in the expression of JARID2 between the 2-cell and 16-cell stages, but expression in the other stages of early bovine embryos was significantly higher than that in the 2-cell stage $(\mathrm{p}<0.05)$. The expression of JARID2 determined based on the fluorescence intensity was consistent with the results of qRT-PCR analysis (Fig. $1 \mathrm{C)}$.

\section{Effect of JARID2 siRNA interference on early embryonic development}

The relative expression of JARID2 mRNA in the bovine cumulus cells after siRNA silencing was detected via qRT-PCR, as shown in Fig. 2. Compared with the negative controls, siRNA2830 had the greatest inhibitory effect on JARID2 - approximately $65 \%(\mathrm{p}<0.05)$. Therefore, siRNA-2830 was used in subsequent experiments (Fig. 2A).

The expression of JARID2 was subsequently knocked down by microinjection of siRNA into the zygotes. After microinjection, we performed IVC, collected embryos at all stages, evaluated embryonic development and conducted statistical analyses of the cleavage rate and blastocyst 
239

240

241

242

243

244

245

246

247

248

249

250

251

252

253

254

255

256

257

258

259

260

261

262

263

264

265

rate. The statistical analyses showed that the cleavage rates were substantially decreased $(\mathrm{p}<0.01)$, and blastocyst rates were significantly decreased $(\mathrm{p}<0.05)$ (Fig. 2B).

\section{Effect of JARID2 on embryonic development-related gene expression}

IVC of zygotes and collection of early embryos at the 2-cell stage and blastula stage, followed by detection of the mRNA expression levels of embryonic development-related genes (OCT4, SOX2 and c-myc), showed that these genes were expressed at higher levels in embryos at the 2cell stage than in embryos at the blastula stage (Fig. 3A). Changes in embryonic developmentrelated gene expression were also detected after JARID2 knockdown in early embryos at the 2cell stage, which showed that the mRNA expression levels of OCT4, SOX2 and c-myc were highly significantly increased (Fig. 3B).

\section{JARID2 knockdown affects histone H3K27 methylation at the 2-cell, 4-cell, 8-cell and blastula stages}

We examined the fluorescence intensity of $\mathrm{H} 3 \mathrm{~K} 27 \mathrm{me} 3$, representing the expression level of H3K27me3, in the early embryos of the JARID2 siRNA silencing group and the negative control group following immunofluorescence staining. The results showed that the expression of H3K27me3 in the JARID2 siRNA silencing group at the 2-cell stage, 4-cell stage and 8-cell stage was significantly lower than that in the control group at the same stages. Additionally, there was no change in H3K27me3 expression in the blastulas of the JARID2 siRNA silencing group compared with the blastulas of the negative control group (Fig. 4A-B).

After microinjection of JARID2 2830 siRNA, the degree of H3K27me3 expression was significantly reduced compared with the controls, but there was no significant difference in H3K27me3 expression at the blastula stage. (Fig. 4C).

\section{DISCUSSION}

JARID2, as a member of the jumonji histone family, has a conserved DNA structural domain 
266

267

268

269

270

271

272

273

274

275

276

277

278

279

280

281

282

283

284

285

286

287

288

289

290

291

292

and could play an important regulatory role in normal early embryonic development as a regulator of several key developmental genes. JARID2 and other members of the jumonji histone family exhibit only $30 \%$ similarity in their spatial structures; therefore, their main functions may be markedly different from other members of the family (Gregory et al. 1996; Iwahara \& Clubb 1999; Takeuchi 1997). Within the histone demethylase family, JARID2 is a special histone that does not show demethylase activity but can elicit histone methyltransferase activity by specific means. JARID2 mainly wraps around EZH2, a member of the PRC2 family, and exerts its methyltransferase activity to affect the maturation rate of oocytes and the cleavage rate of early embryos (Lee \& Skalnik 2005; Zofall \& Grewal 2006). Patients with a chronic malignancy are deficient in the JARID2 protein, which may result in the loss of enzymatic activity during early embryonic development and impede the normal development of embryos (Li et al. 2010; Puda et al. 2012). In this study, through qRT-PCR, immunofluorescence staining and fluorescence intensity analyses, the expression level and pattern of JARID2 were evaluated in bovine oocytes at the GV and MII stages and in bovine embryos at the 2-cell, 4-cell, 8-cell, 16-cell and blastocyst stages. JARID2 was highly expressed in oocytes at the GV and MII stages and in embryos at the 2-cell, 4-cell, 8-cell, 16-cell morula and blastula stages.

JARID2 expression was lower in oocytes at the GV stage than in the other examined stages, and the difference was statistically significant. The above results show that with the development of oocytes and embryos, the levels of JARID2 gene expression increased. These findings indicates that JARID2 may play an important role in the development of early bovine embryos and that embryos develop correctly only in the presence of high levels of JARID2, demonstrating the value of this research.

It has been reported that JARID2 plays an important role in the differentiation of early ESCs as a recruiter of $\mathrm{PRC} 2$, which regulates transcriptional translation primarily through the fine adjustment of chromatin structure to facilitate normal embryonic development (Landeira et al. 2010). JARID2 target sites are rich in CGG and GA sequences (Peng et al. 2009), and the DNA winding sequence irregularly appears in chromatin ( $\mathrm{Li}$ et al. 2010), which is consistent with the 
293 finding of the present study that JARID2 is mainly expressed in the cell nucleus. JARID2

294

295

296

297

298

299

300

301

302

303

304

305

306

307

308

309

310

311

312

313

314

315

316

317

318

319

expression levels represented by the fluorescence intensity were consistent with the results of real-time fluorescent quantitative PCR. After confirming that JARID2 is a nuclear protein, we began to consider what role JARID2 plays in the cell nucleus. JARID2 is important for the development of mouse embryos (Kitajima et al. 1999). Mice lacking the JARID2 protein exhibit developmental deficits, including abnormal development due to excessively fast cell differentiation and abnormal development in cardiomyocytes and megakaryocytes (Jung et al. 2005a; Lee et al. 2000; Mysliwiec et al. 2011; Takeuchi et al. 1999; Takeuchi et al. 1995). In several studies, it was found that overexpression of the JARID2 protein can lead to significantly reduced cell proliferation and DNA synthesis (Toyoda et al. 2000). Our results also demonstrate that JARID2 acts as an important transcription factor or regulatory protein that affects the development of oocytes and embryos, and it may be involved in epigenetic modification.

The results of the present study showed that JARID2-siRNA-2830 could silence JARID2 expression with an interference efficiency of approximately $65 \%(p<0.05)$, based on real-time fluorescent quantitative PCR. Therefore, JARID2-siRNA-2830 could be used in the subsequent experiments. After the knockdown of JARID2 through microinjection of JARID2-2830-siRNA, the cleavage rate and blastocyst rate of early bovine embryos in the JARID2-2830-siRNA silencing group were significantly decreased compared with the negative control group. These results suggest that JARID2 plays an important regulatory role in early bovine embryonic development and that a lack of JARID2 could inhibit the development of early embryos. Several studies have shown that JARID2 is closely related to the production of blastulas during early embryonic development and participates in the formation of organs (Corcoran et al. 2011; Peng et al. 2009; Ulitsky et al. 2011). During germ cell development, JARID2 interacts with most inhibitory complex 2 to regulate the self-renewal ability of ESCs, and the absence of JARID2 reduces the transferability of mouse hematopoietic stem cells and embryonic stem cells (Cai et al. 2013; Margueron et al. 2009; Zhao et al. 2010). Our results are consistent with previous studies. The JARID2 protein plays an indispensable role in early embryonic development. The absence 
320

321

322

323

324

325

326

327

328

329

330

331

332

333

334

335

336

337

338

339

340

341

342

343

344

345

346

of JARID2 hinders normal embryonic development, reducing not only the cleavage rate but also the blastocyst rate. These results suggest that JARID2 promotes early embryonic development. Embryos lacking JARID2 do not develop normally. Based on our research, we can speculate that JARID2 regulates embryonic development by regulating certain factors. Its functions and mechanisms shall be further explored and studied. To further study the mechanism of JARID2 in embryonic development, we examined the changes in embryonic development-related genes and H3K9 methylation after siRNA knockdown of JARID2.

Several papers have reported that the OCT4 gene is mainly expressed in ESCs and reproductive stem cells and plays an important role in maintaining the pluripotency and selfrenewal ability of embryonic stem cells (Looijenga et al. 2003). The OCT4 gene expression level decreases with the differentiation of tissue cells (Hough et al. 2006). SOX2, c-myc and OCT4 have synergistic effects and are important transcription factors that can regulate the pluripotency and self-renewal ability of ESCs; moreover, they play an important role in early embryonic development (Oster et al. 2002). The expression level of SOX2 in early embryos at the 2-cell stage is higher than in early embryos at the blastula stage (Rodda et al. 2005). The expression level of c-myc is decreased after the 8-cell stage, which may be due to the different roles of SOX2 and c-myc in embryonic development. In the present study, JARID2 expression was silenced in zygotes obtained through in vitro fertilization via microinjection of JARID2-2830siRNA. By detecting the mRNA expression levels of OCT4, SOX2 and c-myc at the 2-cell stage with qRT-PCR, we found that the mRNA expression levels of OCT4, SOX2 and c-myc were highly significantly increased in the JARID2 knockdown group compared with the control group. Therefore, we speculate that JARID2 plays an important role in early embryonic development by regulating the expression of OCT4, SOX2 and c-myc.

The members of the jumonji demethylase family exhibit a JmjC structural domain, and most members can demethylate histone lysine using Fe(II) and $\alpha$-ketoglutarate $(\alpha \mathrm{KG})$ as co-enzyme factors. As previously reported, JHDMs can demethylate H3K36 (JHDM1) and H3K9me1; JHDM1 and JHDM2A can demethylate H3K9 and H3K36; and JARID1 can demethylate 
347

348

349

350

351

352

353

354

355

356

357

358

359

360

361

362

363

364

365

366

367

368

369

370

371

372

373

H3K9me3 and H3K27me3. However, whether JARID2, a member of the jumonji protein family, shows catalytic functions or demethylates H3K27me3 is uncertain. Histone H3K27 methylation is an important modification in embryonic development, stem cell formation and tumor transition (Margueron \& Reinberg 2011). PRC2 complexes regulate H3K27 methylation to silence genes (Chase \& Cross 2011), and JARID2 promotes the recruitment of PRC2 to DNA via binding with PRC2 (Li et al. 2010; Pasini et al. 2010; Peng et al. 2009; Shen et al. 2009).

In this study, early embryos were collected at the 2-cell, 4-cell, 8-cell and blastocyst stages after in vitro maturation, in vitro fertilization and microinjection of JARID2-2830-siRNA. Immunofluorescence staining and fluorescence intensity analysis were performed to detect the expression of H3K27me3 after JARID2 interference, and the difference in expression was taken as a measurement index of changes in $\mathrm{H} 3 \mathrm{~K} 27 \mathrm{me} 3$ levels. The experimental results showed that the expression levels of $\mathrm{H} 3 \mathrm{~K} 27 \mathrm{me} 3$ at the 2-cell stage, 4-cell stage and 8-cell stage in the JARID2 knockdown group were highly significantly decreased, whereas the level of H3K27me3 at the blastula stage was not significantly changed. The lack of change in the H3K27me3 level at the blastula stage might be due to the timing of JARID2 siRNA microinjection; after silencing JARID2 at the zygote stage, the silencing efficiency may gradually decrease with embryonic development. It was previously reported that JARID2 interacts with histone methyltransferases at the molecular level or regulates the expression of key genes in embryonic development, thus promoting early embryonic development (Peng et al. 2009; Rinn et al. 2007; Rostovskaya et al. 2012; Son et al. 2013; Yuan et al. 2012). During the process of oocyte meiosis, H3K27me3 may be dynamically changed, which could impact early embryonic development (Kaneko et al. 2014; Kim et al. 2004; Landeira \& Fisher 2011).

\section{CONCLUSION}

This study confirmed that JARID2 is expressed in the cell nucleus of bovine oocytes and early embryos in vitro and that JARID2 interference inhibits embryonic development, reduces the cleavage rate and blastocyst rate and reduces the methylation level of H3K27. In addition, JARID2 could affect early embryonic development by modulating OCT4, SOX and c-myc 
374 expression. Our study results may provide a theoretical basis for discussing the main functions of 375 the interaction between histone lysine methylation and key genes involved in early embryonic 376 development in bovine oocytes and early embryos.

377

378 Data availability

379 The raw data have been supplied as Supplementary Files. 
381

382

383

384

385

386

387

388

389

390

391

392

393

394

395

396

397

398

399

400

401

402

403

404

405

406

407

408

409

410

411

412

413

414

415

416

417

418

419

420

421

\section{REFERENCES}

Brenet F, Moh M, Funk P, Feierstein E, Viale AJ, Socci ND, and Scandura JM. 2011. DNA methylation of the first exon is tightly linked to transcriptional silencing. PLoS One 6:e14524.

Cai L, Rothbart SB, Lu R, Xu B, Chen WY, Tripathy A, Rockowitz S, Zheng D, Patel DJ, Allis CD, Strahl BD, Song J, and Wang GG. 2013. An H3K36 methylation-engaging Tudor motif of polycomb-like proteins mediates PRC2 complex targeting. Mol Cell 49:571-582.

Chase A, and Cross NC. 2011. Aberrations of EZH2 in cancer. Clin Cancer Res 17:2613-2618.

Choy JS, Wei S, Lee JY, Tan S, Chu S, and Lee TH. 2010. DNA methylation increases nucleosome compaction and rigidity. J Am Chem Soc 132:1782-1783.

Corcoran DL, Georgiev S, Mukherjee N, Gottwein E, Skalsky RL, Keene JD, and Ohler U. 2011. PARalyzer: definition of RNA binding sites from PAR-CLIP short-read sequence data. Genome Biol 12:R79.

Cotton AM, Lam L, Affleck JG, Wilson IM, Penaherrera MS, McFadden DE, Kobor MS, Lam WL, Robinson WP, and Brown CJ. 2011. Chromosome-wide DNA methylation analysis predicts human tissue-specific $X$ inactivation. Hum Genet 130:187-201.

Dean W, Lucifero D, and Santos F. 2005. DNA methylation in mammalian development and disease. Birth Defects Res C Embryo Today 75:98-111.

Delcuve GP, Rastegar M, and Davie JR. 2009. Epigenetic control. J Cell Physiol 219:243-250.

Gregory SL, Kortschak RD, Kalionis B, and Saint R. 1996. Characterization of the dead ringer gene identifies a novel, highly conserved family of sequence-specific DNA-binding proteins. Mol Cell Biol 16:792-799.

Herz HM, and Shilatifard A. 2010. The JARID2-PRC2 duality. Genes Dev 24:857-861.

Hou J, Liu L, Zhang J, Cui XH, Yan FX, Guan H, Chen YF, and An XR. 2008. Epigenetic modification of histone 3 at lysine 9 in sheep zygotes and its relationship with DNA methylation. BMC Dev Biol 8:60.

Hough SR, Clements I, Welch PJ, and Wiederholt KA. 2006. Differentiation of mouse embryonic stem cells after RNA interference-mediated silencing of OCT4 and Nanog. Stem Cells 24:1467-1475.

Isagawa T, Nagae G, Shiraki N, Fujita T, Sato N, Ishikawa S, Kume S, and Aburatani H. 2011. DNA methylation profiling of embryonic stem cell differentiation into the three germ layers. PLoS One 6:e26052.

Iwahara J, and Clubb RT. 1999. Solution structure of the DNA binding domain from Dead ringer, a sequencespecific AT-rich interaction domain (ARID). EMBO J 18:6084-6094.

Jackson AL, Bartz SR, Schelter J, Kobayashi SV, Burchard J, Mao M, Li B, Cavet G, and Linsley PS. 2003. Expression profiling reveals off-target gene regulation by RNAi. Nat Biotechnol 21:635-637.

Jung J, Kim TG, Lyons GE, Kim HR, and Lee Y. 2005a. Jumonji regulates cardiomyocyte proliferation via interaction with retinoblastoma protein. J Biol Chem 280:30916-30923.

Jung J, Mysliwiec MR, and Lee Y. 2005b. Roles of JUMONJI in mouse embryonic development. Dev Dyn 232:21-32.

Jurkowska RZ, Jurkowski TP, and Jeltsch A. 2011. Structure and function of mammalian DNA methyltransferases. Chembiochem 12:206-222.

Kaneko S, Bonasio R, Saldana-Meyer R, Yoshida T, Son J, Nishino K, Umezawa A, and Reinberg D. 2014. Interactions between JARID2 and noncoding RNAs regulate PRC2 recruitment to chromatin. Mol Cell 53:290-300.

Kim JM, Liu H, Tazaki M, Nagata M, and Aoki F. 2003a. Changes in histone acetylation during mouse oocyte meiosis. J Cell Biol 162:37-46. 
422

Kim TG, Chen J, Sadoshima J, and Lee Y. 2004. Jumonji represses atrial natriuretic factor gene expression by inhibiting transcriptional activities of cardiac transcription factors. Mol Cell Biol 24:10151-10160.

Kim TG, Kraus JC, Chen J, and Lee Y. 2003b. JUMONJI, a critical factor for cardiac development, functions as a transcriptional repressor. J Biol Chem 278:42247-42255.

Kinkel SA, Galeev R, Flensburg C, Keniry A, Breslin K, Gilan O, Lee S, Liu J, Chen K, Gearing L, Moore DL, Alexander WS, Dawson M, Majewski IJ, Oshlack A, Larsson J, and Blewitt ME. 2015. Jarid2 regulates hematopoietic stem cell function by acting with polycomb repressive complex 2. Blood 125:18901900.

Kitajima K, Kojima M, Nakajima K, Kondo S, Hara T, Miyajima A, and Takeuchi T. 1999. Definitive but not primitive hematopoiesis is impaired in jumonji mutant mice. Blood 93:87-95.

Kooistra SM, and Helin K. 2012. Molecular mechanisms and potential functions of histone demethylases. Nat Rev Mol Cell Biol 13:297-311.

Landeira D, and Fisher AG. 2011. Inactive yet indispensable: the tale of Jarid2. Trends Cell Biol 21:74-80.

Landeira D, Sauer S, Poot R, Dvorkina M, Mazzarella L, Jorgensen HF, Pereira CF, Leleu M, Piccolo FM, Spivakov M, Brookes E, Pombo A, Fisher C, Skarnes WC, Snoek T, Bezstarosti K, Demmers J, Klose RJ, Casanova M, Tavares L, Brockdorff N, Merkenschlager M, and Fisher AG. 2010. Jarid2 is a PRC2 component in embryonic stem cells required for multi-lineage differentiation and recruitment of PRC1 and RNA Polymerase II to developmental regulators. Nat Cell Biol 12:618-624.

Lee JH, and Skalnik DG. 2005. CpG-binding protein (CXXC finger protein 1) is a component of the mammalian Set1 histone H3-Lys4 methyltransferase complex, the analogue of the yeast Set1/COMPASS complex. J Biol Chem 280:41725-41731.

Lee $\mathrm{Y}$, Song AJ, Baker R, Micales B, Conway SJ, and Lyons GE. 2000. Jumonji, a nuclear protein that is necessary for normal heart development. Circ Res 86:932-938.

Li G, Margueron R, Ku M, Chambon P, Bernstein BE, and Reinberg D. 2010. Jarid2 and PRC2, partners in regulating gene expression. Genes Dev 24:368-380.

Looijenga LH, Stoop H, de Leeuw HP, de Gouveia Brazao CA, Gillis AJ, van Roozendaal KE, van Zoelen EJ, Weber RF, Wolffenbuttel KP, van Dekken H, Honecker F, Bokemeyer C, Perlman EJ, Schneider DT, Kononen J, Sauter G, and Oosterhuis JW. 2003. POU5F1 (OCT3/4) identifies cells with pluripotent potential in human germ cell tumors. Cancer Res 63:2244-2250.

Malousi A, Maglaveras N, and Kouidou S. 2008. Intronic CpG content and alternative splicing in human genes containing a single cassette exon. Epigenetics 3:69-73.

Margueron R, Justin N, Ohno K, Sharpe ML, Son J, Drury WJ, 3rd, Voigt P, Martin SR, Taylor WR, De Marco V, Pirrotta V, Reinberg D, and Gamblin SJ. 2009. Role of the polycomb protein EED in the propagation of repressive histone marks. Nature 461:762-767.

Margueron R, and Reinberg D. 2011. The Polycomb complex PRC2 and its mark in life. Nature 469:343-349.

Marinho LSR, Rissi VB, Lindquist AG, Seneda MM, and Bordignon V. 2017. Acetylation and methylation profiles of H3K27 in porcine embryos cultured in vitro. Zygote:1-8.

Mysliwiec MR, Bresnick EH, and Lee Y. 2011. Endothelial Jarid2/Jumonji is required for normal cardiac development and proper Notch1 expression. J Biol Chem 286:17193-17204.

Oster SK, Ho CS, Soucie EL, and Penn LZ. 2002. The myc oncogene: MarvelousIY Complex. Adv Cancer Res 84:81-154. 
Pasini D, Cloos PA, Walfridsson J, Olsson L, Bukowski JP, Johansen JV, Bak M, Tommerup N, Rappsilber J, and Helin K. 2010. JARID2 regulates binding of the Polycomb repressive complex 2 to target genes in ES cells. Nature 464:306-310.

Peng JC, Valouev A, Swigut T, Zhang J, Zhao Y, Sidow A, and Wysocka J. 2009. Jarid2/Jumonji coordinates control of PRC2 enzymatic activity and target gene occupancy in pluripotent cells. Cell 139:1290-1302.

Puda A, Milosevic JD, Berg T, Klampfl T, Harutyunyan AS, Gisslinger B, Rumi E, Pietra D, Malcovati L, Elena C, Doubek M, Steurer M, Tosic N, Pavlovic S, Guglielmelli P, Pieri L, Vannucchi AM, Gisslinger H, Cazzola M, and Kralovics R. 2012. Frequent deletions of JARID2 in leukemic transformation of chronic myeloid malignancies. Am J Hematol 87:245-250.

Rinn JL, Kertesz M, Wang JK, Squazzo SL, Xu X, Brugmann SA, Goodnough LH, Helms JA, Farnham PJ, Segal E, and Chang HY. 2007. Functional demarcation of active and silent chromatin domains in human HOX loci by noncoding RNAs. Cell 129:1311-1323.

Rodda DJ, Chew JL, Lim LH, Loh YH, Wang B, Ng HH, and Robson P. 2005. Transcriptional regulation of nanog by OCT4 and SOX2. J Biol Chem 280:24731-24737.

Ross PJ, Ragina NP, Rodriguez RM, lager AE, Siripattarapravat K, Lopez-Corrales N, and Cibelli JB. 2008. Polycomb gene expression and histone $\mathrm{H} 3$ lysine 27 trimethylation changes during bovine preimplantation development. Reproduction 136:777-785.

Rostovskaya M, Fu J, Obst M, Baer I, Weidlich S, Wang H, Smith AJ, Anastassiadis K, and Stewart AF. 2012. Transposon-mediated BAC transgenesis in human ES cells. Nucleic Acids Res 40:e150.

Shen X, Kim W, Fujiwara Y, Simon MD, Liu Y, Mysliwiec MR, Yuan GC, Lee Y, and Orkin SH. 2009. Jumonji modulates polycomb activity and self-renewal versus differentiation of stem cells. Cell 139:13031314.

Son J, Shen SS, Margueron R, and Reinberg D. 2013. Nucleosome-binding activities within JARID2 and EZH1 regulate the function of PRC2 on chromatin. Genes Dev 27:2663-2677.

Takeuchi T. 1997. A gene trap approach to identify genes that control development. Dev Growth Differ 39:127-134.

Takeuchi T, Kojima M, Nakajima K, and Kondo S. 1999. jumonji gene is essential for the neurulation and cardiac development of mouse embryos with a C3H/He background. Mech Dev 86:29-38.

Takeuchi T, Yamazaki Y, Katoh-Fukui Y, Tsuchiya R, Kondo S, Motoyama J, and Higashinakagawa T. 1995. Gene trap capture of a novel mouse gene, jumonji, required for neural tube formation. Genes Dev 9:12111222.

Toyoda M, Kojima M, and Takeuchi T. 2000. Jumonji is a nuclear protein that participates in the negative regulation of cell growth. Biochem Biophys Res Commun 274:332-336.

Ulitsky I, Shkumatava A, Jan CH, Sive H, and Bartel DP. 2011. Conserved function of lincRNAs in vertebrate embryonic development despite rapid sequence evolution. Cell 147:1537-1550.

Verona RI, Mann MR, and Bartolomei MS. 2003. Genomic imprinting: intricacies of epigenetic regulation in clusters. Annu Rev Cell Dev Biol 19:237-259.

Walsh CP, Chaillet JR, and Bestor TH. 1998. Transcription of IAP endogenous retroviruses is constrained by cytosine methylation. Nat Genet 20:116-117.

Yeo S, Lee KK, Han YM, and Kang YK. 2005. Methylation changes of lysine 9 of histone H3 during preimplantation mouse development. Mol Cells 20:423-428. 
504

505

506

507

508

509

510

511

512

513

514
Yuan W, Wu T, Fu H, Dai C, Wu H, Liu N, Li X, Xu M, Zhang Z, Niu T, Han Z, Chai J, Zhou XJ, Gao S, and Zhu B. 2012. Dense chromatin activates Polycomb repressive complex 2 to regulate H3 lysine 27 methylation. Science 337:971-975.

Zhang M, Wang F, Kou Z, Zhang Y, and Gao S. 2009. Defective chromatin structure in somatic cell cloned mouse embryos. J Biol Chem 284:24981-24987.

Zhao J, Ohsumi TK, Kung JT, Ogawa Y, Grau DJ, Sarma K, Song JJ, Kingston RE, Borowsky M, and Lee JT. 2010. Genome-wide identification of polycomb-associated RNAs by RIP-seq. Mol Cell 40:939-953.

Zofall M, and Grewal SI. 2006. Swi6/HP1 recruits a JmjC domain protein to facilitate transcription of heterochromatic repeats. Mol Cell 22:681-692. 


\section{FIGURE LEGENDS}

Fig. 1 Relative expression of JARID2 in bovine oocytes and early embryos.

A: Relative expression of JARID2 mRNA in oocytes at the GV stage and embryos at the 2-cell, 4-cell, 8-cell, 16-cell and blastula stages. JARID2 is expressed at all oocyte and early embryonic stages, and its expression at the MII stage and early embryonic stages is significantly higher than that at the GV stage $(\mathrm{p}<0.05)$.

B: Immunofluorescence localization of JARID2 protein in early embryonic development. In A1 E1, green FITC fluorescence represents the location of the JARID2 protein at the 2-cell, 4cell, 8-cell, 16-cell and blastula stages, and in A2 E2 blue DAPI fluorescence represents the location of DNA in the cell nucleus. A3 E3 are the merged images.

$\mathrm{C}$ : Immunofluorescence intensity of the JARID2 protein in early embryonic development. The results show that JARID2 is expressed at all stages of early embryonic development.

Data are presented as the mean \pm SD derived from triplicate transfectants in three independent experiments.

Different superscripts ( $a$ and $b$ ) denote a statistically significant difference.

* denotes a statistically significant difference $(\mathrm{p}<0.05)$.

Fig. 2 Influence of JARID2 siRNA interference on early embryonic development.

A: Silencing effects of siRNA targeting different loci in JARID2 in bovine cumulus cells. Among the three siRNAs, siRNA-2830 showed the highest silencing efficiency. After microinjection, the expression of JARID2 was significantly lower in the siRNA-2830-injected group by approximately $65 \%$ than in the negative control group $(\mathrm{p}<0.05)$; therefore, siRNA-2830 was suitable for subsequent RNA interference.

B: Effect of JARID2 silencing through microinjection of siRNA-2830 on early embryonic development. The cleavage rate was highly significantly lower $(p<0.01)$, and the blastocyst rate was significantly lower $(\mathrm{p}<0.05)$ in the siRNA-treated group than in the negative control group. Data are presented as the mean \pm SD derived from triplicate transfectants in three independent experiments.

Different superscripts ( $a$ and $b$ ) denote a statistically significant difference.

* denotes a statistically significant difference $(\mathrm{p}<0.05)$, and $* *$ denotes an extremely significant difference $(\mathrm{p}<0.01)$.

Fig. 3 Effect of JARID2 on embryonic development-related gene expression.

A: Relative mRNA expression of OCT4, SOX2 and c-myc in embryos at the 2-cell and blastocyst stages. At the blastocyst stage, the expression of the three genes was highly significantly lower than that at the 2-cell stage $(\mathrm{p}<0.01)$.

B: Relative expression of embryonic development-related genes after transfection of siRNA- 
5552830 to knock down JARID2 expression. JARID2 expression was highly significantly reduced $556(\mathrm{p}<0.01)$, and OCT4, SOX2 and c-myc expression was highly significantly increased $(\mathrm{p}<0.01)$ in 557 the siRNA-2830-transfected group compared with the control group.

558 Data are presented as the mean \pm SD derived from triplicate transfectants of three independent 559 experiments.

$560 * *$ denotes an extremely significant difference $(\mathrm{p}<0.01)$.

Fig. 4 JARID2 knockdown affects histone H3K27 methylation at the 2-cell, 4-cell, 8-cell and blastula stages. A: H3K27me3 immunofluorescence staining in the negative control group; green FITC fluorescence represents $\mathrm{H} 3 \mathrm{~K} 27 \mathrm{me} 3$ expression, and blue DAPI fluorescence represents the location of DNA in the cell nucleus.

B: H3K27me3 immunofluorescence staining in the JARID2 knockdown group. C: Immunofluorescence intensity analysis. H3K27 methylation in the knockdown group was highly significantly lower at the 2-cell and 4-cell stages than that in the negative control group at the same stages $(\mathrm{p}<0.01)$, and H3K27 methylation at the 8-cell stage was significantly lower $(\mathrm{p}<0.05)$ than that in the control group; however, at the blastula stage, H3K27 methylation was not significantly different between the two groups $(\mathrm{p}>0.05)$. Data are presented as the mean \pm SD derived from triplicate transfectants of three independent experiments.

$576 *$ denotes a statistically significant difference $(\mathrm{p}<0.05)$, and $* *$ denotes an extremely significant 577 difference $(\mathrm{p}<0.01)$. 


\section{Figure 1}

Relative expression of JARID2 in bovine oocytes and early embryos.

A: Relative expression of JARID2 mRNA in oocytes at the GV stage and embryos at the 2-cell, 4-cell, 8-cell, 16-cell and blastula stages. JARID2 is expressed at all oocyte and early embryonic stages, and its expression at the M2 stage and early embryonic stages is significantly higher than that at the GV stage $(p<0.05)$. B: Immunofluorescence localization of JARID2 protein in early embryonic development. In A1 E1, green FITC fluorescence represents the location of JARID2 protein at the 2-cell, 4-cell, 8-cell, 16-cell and blastula stages, and A2 E2 blue DAPI fluorescence represents the location of DNA in the cellnucleus. A3 E3 are the merged images. C: The immunofluorescence intensity of JARID2 protein in early embryonic development. The results show that JARID2 is expressed at all stages of early embryonic development. Data are presented as the mean \pm SD derived from triplicate transfectants of three independent experiments. Different superscripts ( $a$ and $b$ ) denote a statistically significant difference. $*$ denotes a statistically significant difference $(p<0.05)$. 
A

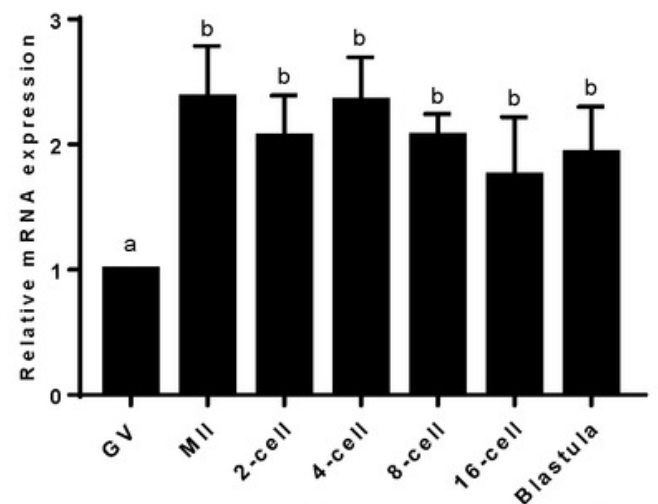

B
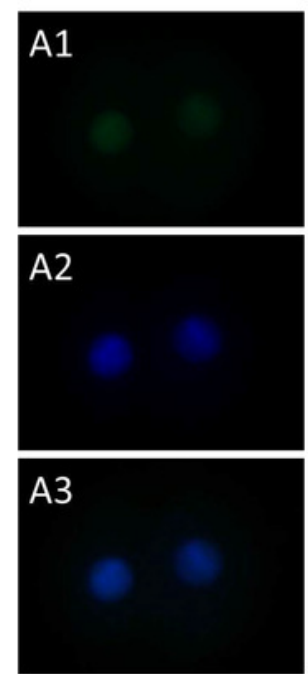

C1

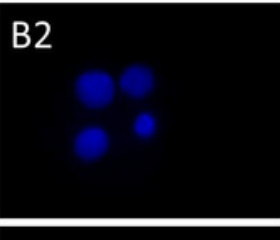

B2

B3

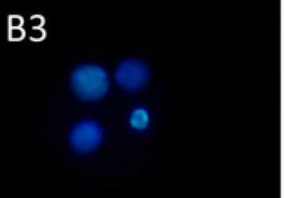

C2

C3
C

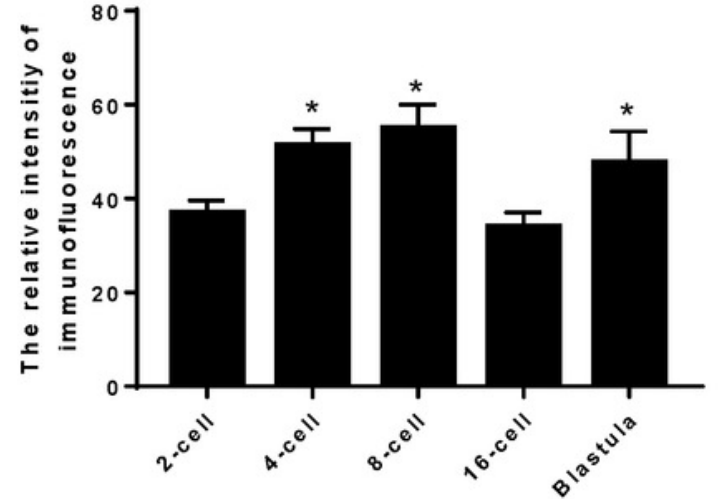

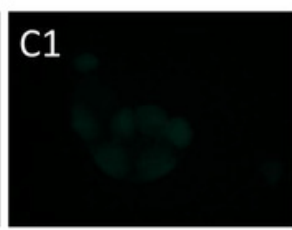

D1

D3

E3 


\section{Figure 2}

The influence of JARID2 siRNA interference on early embryonic development.

A: The silencing effects of siRNA targeting different loci on JARID2 in bovine cumulus cell. Among the three siRNAs, siRNA-2830 had the highest silencing efficiency. After microinjection, the expression of JARID2 was significantly lower in the siRNA-2830-injected group by about $65 \%$ than the negative control group $(p<0.05)$; therefore, siRNA-2830 was suitable for subsequent RNA interference. B: The effect of JARID2 silencing through microinjection of siRNA-2830 on early embryonic development. The cleavage rate were extremely significantly lower $(p<0.01)$ and blastocyst rate were significantly lower $(p<0.05)$ in the siRNA-treated group than in the negative control group. Data are presented as the mean $\pm S D$ derived from triplicate transfectants of three independent experiments. Different superscripts ( $a$ and $b$ ) denote a statistically significant difference. * denotes a statistically significant difference $(p<0.05)$, and ${ }^{* *}$ denotes an extremely significant difference $(p<0.01)$.

A

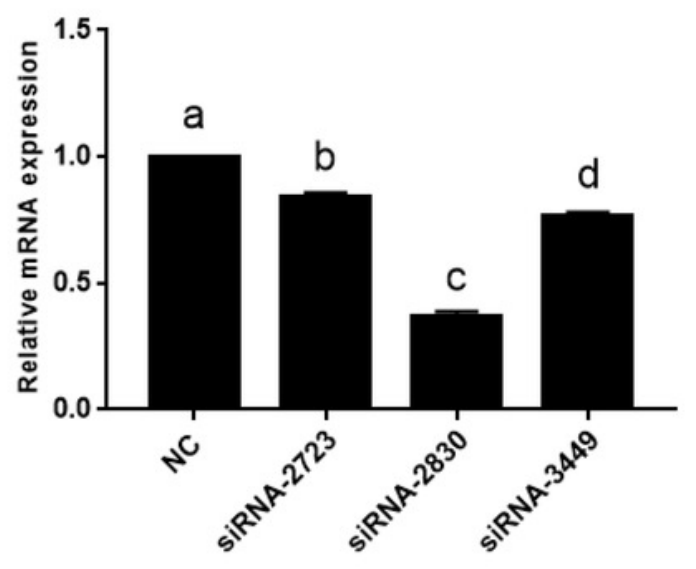

B

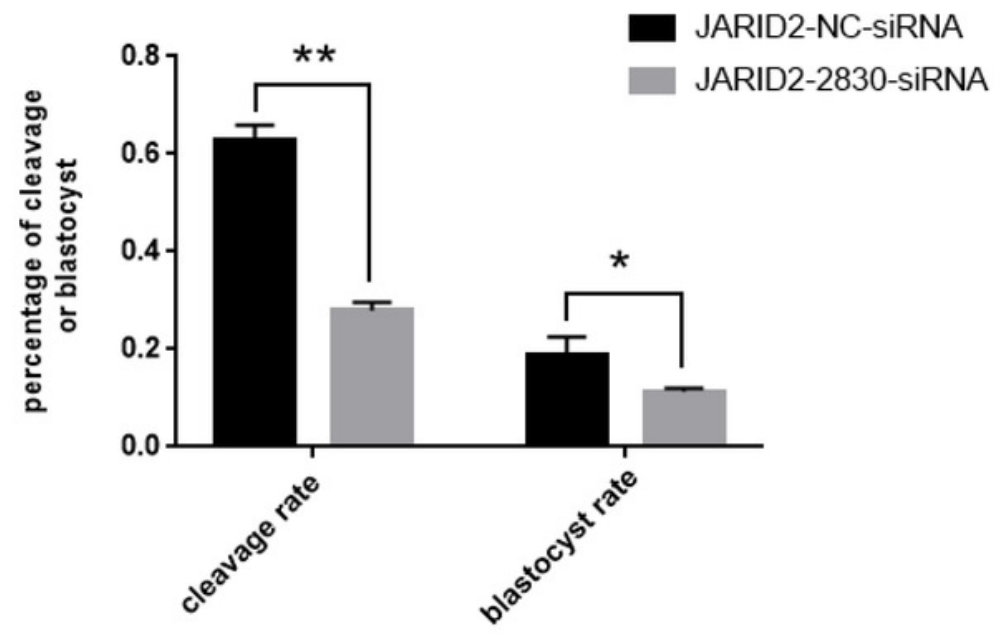




\section{Figure 3}

The effect of JARID2 on embryonic development-related gene expression.

A: Relative mRNA expression of OCT4, SOX2 and c-myc in embryos at the 2-cell and blastocyst stages. At the blastocyst stage, expression of the three genes was significantly lower than that at the 2 -cell stage $(p<0.05)$. B: Relative expression of embryonic development-related genes after transfection of siRNA-2830 to knockdown JARID2 expression. JARID2 expression was significantly reduced $(p<0.05)$ and OCT4, SOX2 and cmyc expression was extremely significantly increased in the siRNA-2830-transfected group compared with the control group $(p<0.01)$. Data are presented as the mean $\pm S D$ derived from triplicate transfectants of three independent experiments. * denotes a statistically significant difference $(p<0.05)$, and $* *$ denotes an extremely significant difference $(p<0.01)$.

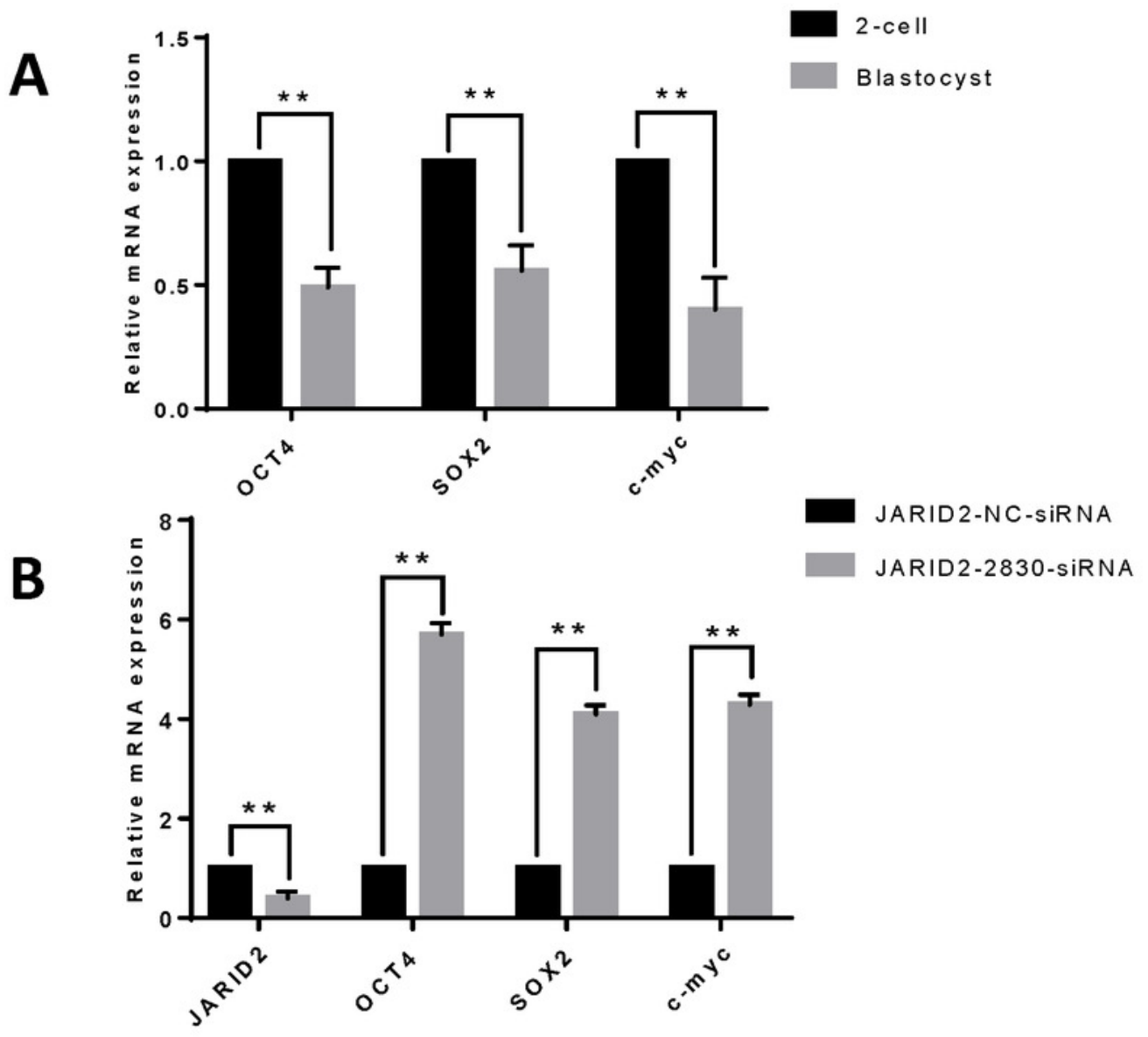




\section{Figure 4}

JARID2 knockdown affects histone H3K27 methylation at the 2-cell, 4-cell, 8-cell and blastula stages.

A: H3K27me3 immunofluorescence staining in the negative control group; green FITC fluorescence represents H3K27me3 expression, and blue DAPI fluorescence represents the location of DNA in the cellnucleus. B: H3K27me3 immunofluorescence staining in the JARID2 knockdown group. C: Immunofluorescence intensity analysis. H3K27 methylation of the knockdown group at the 2-cell, 4-cell and 8-cell stages was significantly lower than that of the negative control group at the same stages $(p<0.05)$; however, at the blastula stage, H3K27 methylation was not significantly different between the two groups ( $p>0.05)$. Data are presented as the mean \pm SD derived from triplicate transfectants of three independent experiments. * denotes a statistically significant difference $(p<0.05)$, and $* *$ denotes an extremely significant 585 difference $(p<0.01)$. 

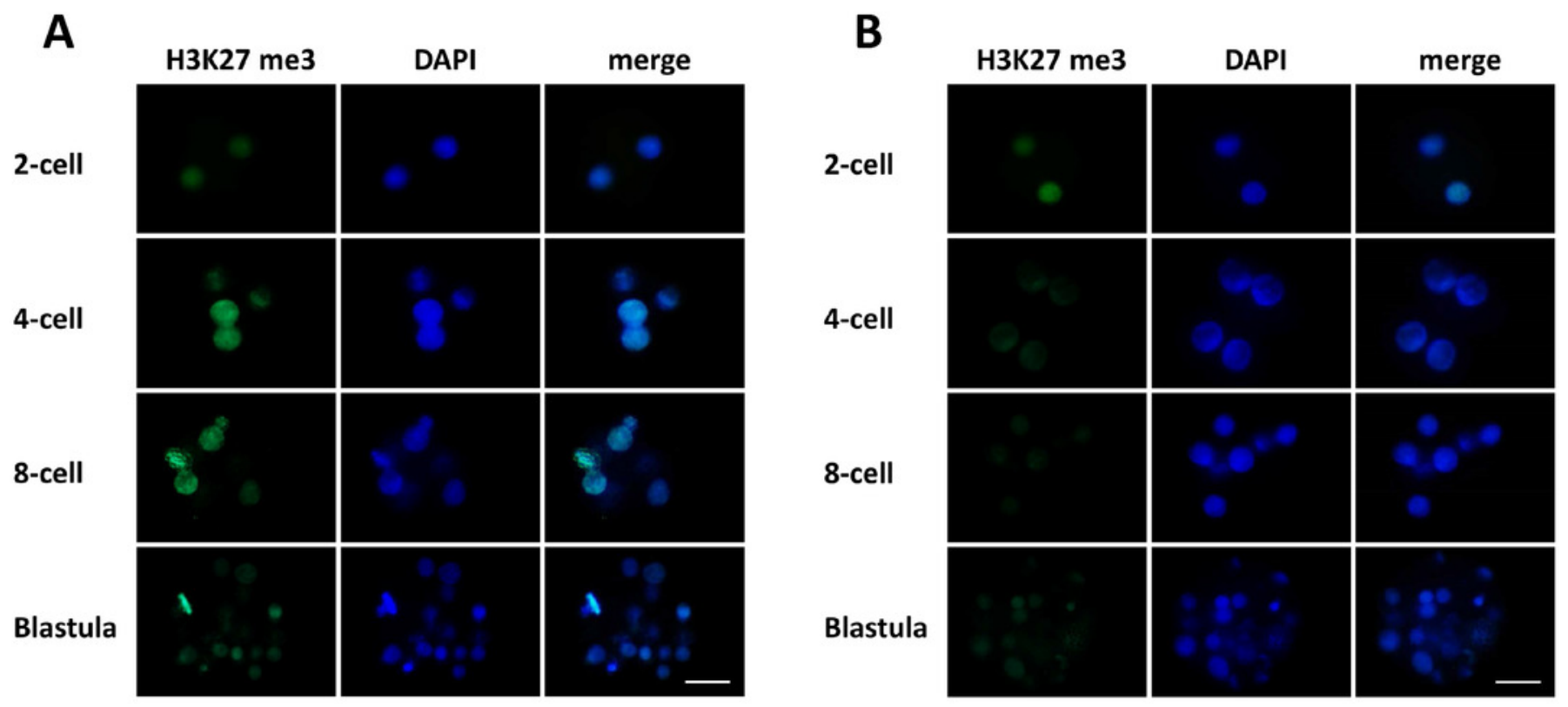

C

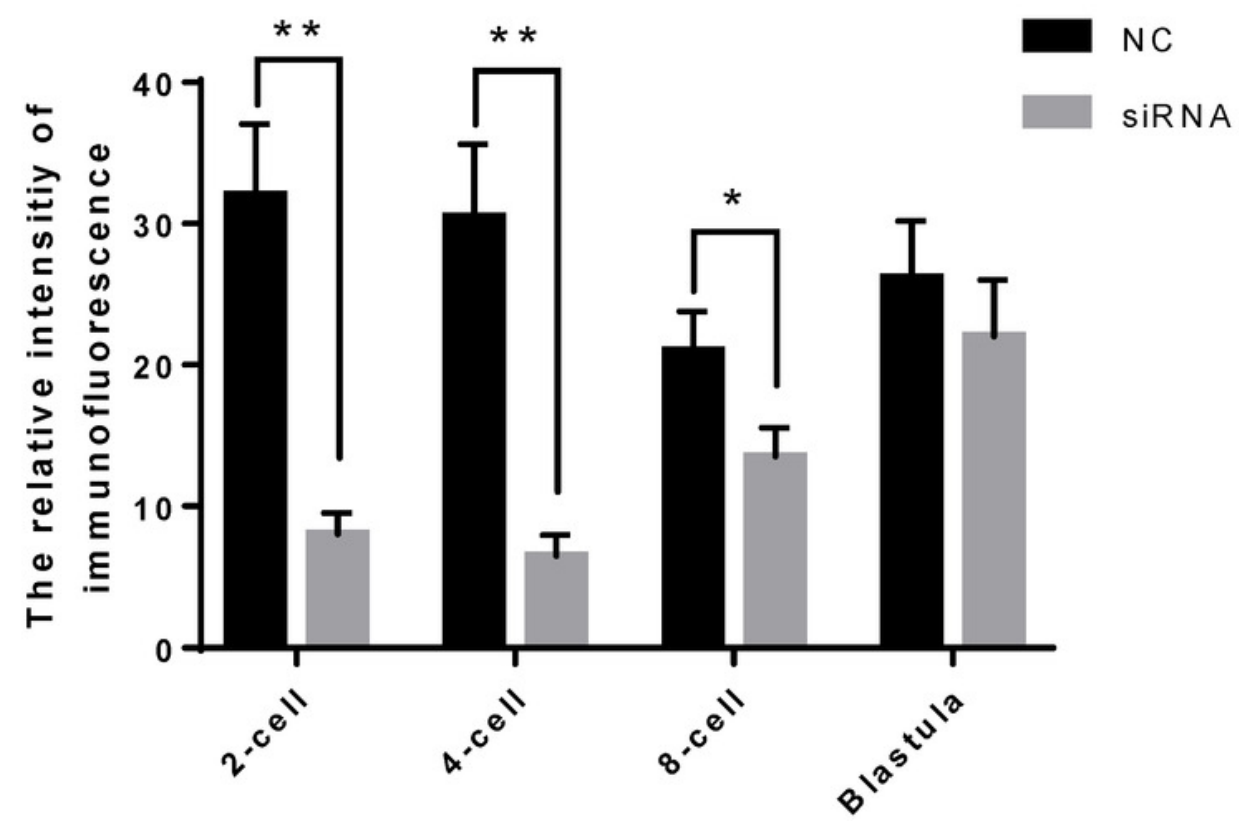

